\section{Condição socioeconômica, frequência de escovação dentária e comportamentos em saúde em adolescentes brasileiros: uma análise a
partir da Pesquisa Nacional de Saúde do Escolar
(PeNSE)}

\author{
Socioeconomic status, toothbrushing frequency, \\ and health-related behaviors in adolescents: \\ an analysis using the PeNSE database
}

\author{
${ }^{1}$ Instituto de Estudos de \\ Saúde Coletiva, Universidade \\ Federal do Rio de Janeiro, \\ Rio de Janeiro, Brasil. \\ 2 Pontifícia Universidade \\ Católica do Paraná, Curitiba, \\ Brasil. \\ ${ }^{3}$ Secretaria de Vigilância em \\ Saúde, Ministério da Saúde, \\ Brasília, Brasil. \\ 4 Universidade Federal do Rio \\ Grande do Sul, Porto Alegre, \\ Brasil. \\ Correspondência \\ M. V. Vettore \\ Instituto de Estudos de Saúde \\ Coletiva, Universidade Federal \\ do Rio de Janeiro. \\ Praça Jorge Machado Moreira, \\ Ilha do Fundão, Cidade \\ Universitária, Rio de Janeiro, \\ RJ 21944-970, Brasil. \\ mario@ensp.fiocruz.br
}

\section{Abstract}

This study investigated the association between oral and general health-related behaviors and socioeconomic status, and the relationship between health-related behaviors and toothbrushing among adolescents. The database used here was the National School-Based Health Survey (PeNSE), a cross-sectional population-based study in 2009 with students from 27 Brazilian State capitals. Socio-demographic and healthrelated behavior data were collected. The survey included 49,189 adolescents (47.5\% males), the majority of whom were 14 years of age and enrolled in public schools. The associations between toothbrushing frequency and other health-related behaviors and socioeconomic status varied between boys and girls. Associations were observed between health-related habits and toothbrushing frequency in both sexes, but with variations according to socioeconomic status. Planning health promotion interventions for adolescents should take their individual characteristics and family and social context into account.

Adolescent Behavior; Toothbrushing; Oral Hygiene; Oral Health
Mario Vianna Vettore 1

Samuel Jorge Moysés 2

Luciana Monteiro Vasconcelos Sardinha 3,4

Betine Pinto Moehlecke Iser 3,4

\section{Introdução}

A adolescência é um período crítico para a saúde, pois durante a transição da infância para a vida adulta, os indivíduos experimentam importantes mudanças biológicas, cognitivas, emocionais e sociais. Esta fase é um importante momento para adoção de novas práticas e comportamentos, ganho de autonomia, exposição a diversas situações e riscos presentes e futuros para a saúde geral e bucal. A exposição de adolescentes a fatores de risco sociocomportamentais, como o tabagismo e o consumo de álcool, a violência, a delinquência escolar e a experimentação de drogas ilícitas, práticas sexuais inseguras, alimentação inadequada e sedentarismo tem sido um achado comum na literatura $1,2,3$.

Os adolescentes, tal qual a população em geral, comportam-se não apenas movidos por suas capacidades e escolhas individuais, mas também são condicionados por determinantes sociais, tais como renda e educação, capazes de definir gradientes e retratar iniquidades em saúde geral e bucal, com impacto imediato ou futuro 4,5,6,7,8. Por outro lado, comportamentos individuais comprometedores em relação à saúde, combinados com uma pobre autopercepção de saúde, na adolescência, predizem um nível educacional mais baixo na maioridade, influenciando negativamente a inserção profissional, ocupação e renda 9. 
Estudos em contextos geopopulacionais diversificados têm identificado aspectos multidimensionais relacionados com a saúde de adolescentes, sugerindo que fatores de risco individuais e determinantes sociais podem revelar associações importantes para a saúde geral e bucal deste grupo etário. Torna-se relevante observar desde fatores sociodemográficos e psicossociais, tais como as condições socioeconômicas dos pais, o desempenho e o seriamento escolar, a qualidade da escola, até fatores grupais e individuais tais como estilos de vida compartilhados entre amigos e comportamentos que mimetizam o grupo, bem como o uso de serviços odontológicos 10,11,12,13,14.

Paralelamente, pesquisas realçam a importância do ambiente social em estágios precoces da vida dos adolescentes, com ênfase para a escolaridade e empregabilidade materna, bem como a situação de saúde bucal precoce e tardia, impactando a qualidade de vida do adolescente independentemente da renda familiar e uso de serviços odontológicos 15,16.

A população jovem é um grupo prioritário para políticas de promoção da saúde em todas as regiões do mundo e a escola se constitui em um espaço privilegiado para implementação destas políticas 17. O Brasil tem cerca de 17,5 milhões de adolescentes (9,2\% da população) entre 10 e 14 anos de idade e 17 milhões (8,9\%) entre 15 e 19 anos ${ }^{18}$. A grande maioria destes adolescentes está na escola, com $98 \%$ e $84 \%$ para as faixas de 10 a 14 e 15 a 17 anos, respectivamente 18.

A Organização Mundial da Saúde (OMS) preconiza o monitoramento da saúde do escolar e desenvolveu, em colaboração com o Centers for Disease Control and Prevention (CDC), dos Estados Unidos, uma metodologia para esta finalidade, o Global School-based Student Health Survey (GSHS) 19. Esta estratégia, que investiga fatores de risco comportamentais e de proteção em adolescentes de 13 a 15 anos, foi implantada ou está em processo de implantação em 22 países. $\mathrm{O}$ Youth Health Risk Behavior Surveillance System (YRBSS) é realizado nos Estados Unidos a cada dois anos desde 1990, pelo CDC, investigando escolares de 12 a 21 anos de idade (http://www. cdc.gov/HealthyYouth/yrbs/index.htm, acessado em 15/Nov/2010). O Health Behaviour in School-aged Children Study (HBSC) é um sistema de monitoramento iniciado na Finlândia, Noruega e Inglaterra em 1982, e atualmente é realizado em outros países da Europa em cooperação com a OMS. Estuda escolares de 11 a 15 anos de idade e também investiga comportamentos de risco relacionados com a saúde 20.

No Brasil, a Pesquisa Nacional de Saúde do Escolar (PeNSE) se constituiu no primeiro inquérito nacional em escolares, atendendo aos objetivos de implantação do Sistema Nacional de Monitoramento da Saúde do Escolar. Visa subsidiar gestores e profissionais de saúde com informações confiáveis para a orientação e avaliação de um conjunto de políticas de saúde destinadas aos adolescentes. Na PeNSE foram coletadas informações sociodemográficas, sobre alimentação, imagem corporal, atividade física, tabagismo, consumo de álcool e outras drogas, saúde bucal, comportamento sexual, violência, acidentes, segurança e medidas antropométricas. O módulo de saúde bucal foi composto por duas questões, referentes à frequência de escovação dentária e relato de dor dentária 21.

O presente estudo teve por objetivo descrever comportamentos relacionados à saúde bucal e geral de adolescentes, segundo sexo e condição socioeconômica. Além disso, testar a associação entre comportamentos relacionados à saúde bucal e geral e condição socioeconômica, e avaliar a associação entre comportamentos relacionados à saúde e escovação dentária segundo condição socioeconômica.

\section{Métodos}

A PeNSE foi um estudo transversal, de base populacional, realizado no período de março a junho de 2009. A população do estudo foi constituída por alunos do 9o ano do Ensino Fundamental, de escolas públicas e privadas das 27 capitais brasileiras, incluindo o Distrito Federal. O trabalho foi coordenado pelo Ministério da Saúde em parceria com o Instituto Brasileiro de Geografia e Estatística (IBGE), responsável pelo trabalho de campo. A escolha do 9o ano do Ensino Fundamental deve-se ao nível mínimo de escolarização necessária para responder o questionário autoaplicável utilizado, e também pela proximidade da idade de referência preconizada pela OMS, que é de 13 a 15 anos 21.

As informações para o cálculo da amostra foram provenientes do Censo Escolar, realizado pelo Instituto Nacional de Estudos e Pesquisas Educacionais Anísio Teixeira (INEP), do Ministério da Educação, em 2007. A amostra foi composta por amostragem probabilística em dois estágios, tendo como Unidade Primária de Amostragem (UPA) as escolas e Unidade Secundária de Amostragem (USA) as turmas de $9^{\circ}$ ano das escolas selecionadas. A amostra de cada estrato foi alocada proporcionalmente ao número de escolas segundo dependência administrativa - pública e privada. O cadastro de seleção da amostra foi constituído por 6.780 escolas que informaram possuir turmas do 9o ano do Ensino Fundamen- 
tal, com no mínimo 15 estudantes na série. As escolas selecionadas no primeiro estágio foram visitadas para construção de uma lista atualizada de turmas existentes em 2009, no seriamento de interesse do estudo, para então selecionar as turmas. O tamanho da amostra foi calculado em cada um dos 27 estratos geográficos de modo a estimar prevalências de $50 \%$, com um erro da ordem de três pontos percentuais, e nível de significância de cinco pontos percentuais 18 .

Foram incluídos na amostra todos os estudantes da turma presentes no dia da coleta de dados que aceitaram voluntariamente participar do estudo. As perdas por ausência ou recusa foram contabilizadas para posterior análise. Também foram excluídos da análise os estudantes que não responderam à variável sexo do questionário.

A coleta de dados foi realizada por meio de microcomputador de mão, Personal Digital Assistant (PDA), contendo questionário estruturado e autoaplicável dividido em módulos por assunto. As características sociodemográficas coletadas foram idade, sexo, raça/cor da pele autodeclarada, tipo de escola (pública ou privada), escolaridade da mãe, residir com o pai e residir com a mãe. A escolaridade materna foi empregada para caracterizar a condição socioeconômica dos adolescentes, e a variável foi agrupada em 4 categorias: sem estudo, Ensino Fundamental completo, Ensino Médio completo e Ensino Superior completo. Nas situações onde a mãe do escolar apresentou-se com a formação incompleta, esta foi alocada na categoria inferior. Por exemplo, mães com Ensino Médio incompleto foram categorizadas como Ensino Fundamental completo.

Comportamentos relacionados à saúde bucal e geral foram mensurados com escalas de 5 pontos, gerando as categorias que podem ser observadas nas respectivas tabelas, considerando: a frequência diária de escovação dentária, número de dias que comeu doces nos últimos 7 dias, número de dias que fumou nos últimos 30 dias, número de dias que tomou pelo menos um copo ou dose de bebida alcoólica nos últimos 30 dias, número de dias que usou drogas nos últimos 30 dias, número de dias que fez atividade física fora da escola nos últimos 7 dias e frequência do uso de cinto de segurança.

\section{Análise dos dados}

Inicialmente foram estimadas as frequências ponderadas e os respectivos intervalos de confiança com precisão de 95\% (IC95\%) para as características sociodemográficas e comportamentos relacionados à saúde bucal e geral, segundo sexo. Posteriormente, as variáveis relacionadas aos comportamentos em saúde bucal e geral foram dicotomizadas para fins de comparação com as categorias de escolaridade materna.

Duas análises de regressão logística com a estimação de odds ratio e respectivos IC95\% foram realizadas. Na primeira análise a variável dependente foi condição socioeconômica e na segunda, frequência de escovação dentária. Em ambas as análises as variáveis independentes foram os comportamentos relacionados à saúde, e foram estratificadas por sexo e condição socioeconômica. A frequência de escovação dentária foi dicotomizada em menos de 2 vezes ao dia e 2 vezes ou mais por dia. Não ignoramos o debate científico que se trava em relação ao uso de regressão logística, para estudos com desfecho comum (usualmente > 10\%) 22. Questiona-se que a odds ratio obtida tende a produzir vieses nas medidas de associação analisadas. Optamos, contudo, pelo uso de regressão logística tendo em vista a simplicidade interpretativa e porque não há modificação na hierarquia, significância e direção das associações observadas, sendo as mudanças de magnitude irrelevantes para a saúde pública. Todas as análises foram realizadas com o uso do programa SPSS, versão 17.0 (SPSS Inc., Chicago, Estados Unidos), com o emprego do módulo complex samples e de um arquivo "csaplan" utilizados para corrigir o efeito do desenho e contemplar os pesos amostrais.

\section{Considerações éticas}

A PeNSE foi aprovada pela Comissão Nacional de Ética em Pesquisa, Conselho Nacional de Saúde, do Ministério da Saúde, sob o parecer no. 11.537. Todas as informações tanto do aluno quanto da escola foram confidenciais e não identificadas.

\section{Resultados}

Este estudo foi conduzido em 2.175 turmas de 1.453 escolas selecionadas que tinham alunos no 9o ano do Ensino Fundamental. Nestas turmas havia 72.872 alunos matriculados e 68.735 alunos que efetivamente frequentavam a escola. No dia da coleta 63.411 alunos estavam nas escolas (92,3\%). Destes, 501 se recusaram a participar da pesquisa (taxa de não resposta de 0,8\%) e 1.937 foram inicialmente excluídos porque não preencheram a variável sexo. Dentre os 60.973 alunos que responderam ao questionário, foram excluídos os que não informaram ( $\mathrm{n}=938$ ) ou que não sabiam a escolaridade da mãe $(n=10.253)$, além dos que não informaram o tipo de escola ( $n=659$ ), perfazendo uma perda de $21,8 \%$ e uma amostra final de 49.189 adolescentes. 
A amostra foi composta por $47,5 \%$ de adolescentes do sexo masculino (IC95\%: 46,8-48,2) e 52,5\% do sexo feminino (IC95\%: 51,8-53,2). A idade/faixa etária predominante para ambos os sexos foi a de 14 anos $(47,1 \%)$, seguida de $\leq 13$ anos $(24,4 \%)$. As categorias de raça/cor da pele branca $(40,1 \%)$ e parda $(39,1 \%)$ foram as mais observadas, sendo a branca estatisticamente mais prevalente no sexo masculino $(41,6 \%)$ e a parda no feminino (42,3\%). A maioria dos entrevistados estudava em escola pública $(78,9 \%)$ e morava com a mãe $(90,6 \%)$, padrão semelhante entre os sexos. A proporção de adolescentes do sexo masculino que morava com o pai $(66,1 \%)$ foi estatisticamente maior em relação ao sexo feminino (60,5\%). A prevalência de adolescentes com mães sem estudo $(31,8 \%)$ foi semelhante àquela de adolescentes com mães com Ensino Médio completo (31,5\%), seguida por aqueles com mães com Ensino Superior (19,8\%) e Ensino Fundamental completo (16,9\%). A escolaridade materna foi estatisticamente maior para o sexo masculino, em que mães com Ensino Médio e Superior completos corresponderam a 53,9\%, enquanto no sexo feminino esta proporção correspondeu a $49,1 \%$ (Tabela 1).

A distribuição dos adolescentes de acordo com os comportamentos relacionados à saúde segundo o sexo é apresenta na Tabela 2. A frequência de escovação dentária duas vezes ou mais ao dia foi de $95,2 \%$, sendo esta maior para as adolescentes do sexo feminino. O consumo diário de doces nos últimos sete dias foi de $34,5 \%$. Adolescentes do sexo feminino reportaram um consumo diário de doces estatisticamente maior do que do sexo masculino (41,5 versus $26,8 \%$ ). A frequência de tabagismo, uso de bebida alcoólica e drogas nos últimos 30 dias foi de $6,3 \%, 27,3 \%$ e 3,3\%, observando-se que, enquanto a ocorrência de tabagismo não diferiu entre os sexos, uso de bebida alcoólica e drogas foram hábitos estatisticamente mais comuns no sexo masculino. A prática de atividade física fora da escola na última semana foi relatada por $56,9 \%$ da amostra, e foi estatisticamente maior em adolescentes do sexo masculino em relação ao feminino (66,7 versus $48,1 \%)$. A maioria dos entrevistados $(62,7 \%)$ informou que usou cinto de segurança sempre ou na maioria das vezes no carro nos últimos 30 dias. Apesar de ambos os sexos apresentarem uso semelhante do cinto de segurança, usar sempre o cinto de segurança foi estatisticamente mais frequente em adolescentes do sexo masculino $(50,1 \%)$ em relação ao feminino (45,8\%).

A caracterização dos comportamentos relacionados à saúde dos adolescentes conforme a condição socioeconômica, representada pela escolaridade materna, para os sexos masculino e feminino, é apresentada nas Tabelas 3 e 4 . Em adolescentes do sexo masculino, a frequência de escovação dentária e o consumo de doces aumentaram com a escolaridade materna, enquanto o tabagismo diminuiu, porém sem diferenças estatisticamente significantes (Tabela 3). Para o sexo feminino, a frequência de escovação dentária aumentou com a escolaridade materna, e a ingestão de doces em três ou mais dias da semana foi relatada por mais de $80 \%$ da amostra em todos os grupos de escolaridade materna. O tabagismo diminuiu conforme o aumento da escolaridade materna, sendo estatisticamente maior entre aquelas com mães com Ensino Médio em relação àquelas com Ensino Superior (Tabela 4). O consumo de bebidas alcoólicas e de drogas ilícitas não demonstrou qualquer tendência com o aumento da escolaridade materna em adolescentes de ambos os sexos. Por outro lado, a prática de atividade física fora da escola e o uso do cinto de segurança aumentaram com a escolaridade materna (Tabelas 3 e 4).

A maior frequência de escovação dentária foi inversamente associada com a menor escolaridade materna, tanto no sexo masculino $(\mathrm{OR}=0,73$; IC95\%: 0,55-0,96) quanto no feminino ( $\mathrm{OR}=0,62$; IC95\%: 0,45-0,87). A ingestão de doces não se associou com a escolaridade materna no sexo masculino. As adolescentes com mães com Ensino Médio tiveram uma chance $20 \%$ maior de comerem mais doces em relação àquelas com Ensino Superior. Todavia, aquelas com mães sem estudo apresentaram uma probabilidade menor de comerem doce quando comparadas àquelas com mães com maior escolaridade (OR $=0,82$; IC95\%: 0,70-0,96). O tabagismo foi estatisticamente associado com falta de escolaridade materna no sexo masculino. Além disso, observou-se um gradiente entre a menor escolaridade materna e a maior chance de tabagismo entre as adolescentes. Escolares de ambos os sexos, com mães com Ensino Fundamental, apresentaram uma menor probabilidade de ingestão de bebidas alcoólicas em comparação com aqueles cujas mães têm Ensino Superior. Enquanto o uso de drogas não se associou com a escolaridade materna, a maior frequência de atividades físicas fora da escola e do uso do cinto de segurança foram inversamente associados com a menor escolaridade materna (Tabela 5).

Na Tabela 6 são apresentados os resultados da associação entre comportamentos relacionados à saúde com a frequência de escovação dentária, segundo as categorias de escolaridade materna e sexo. A ingestão de doces foi estatisticamente associada com a escovação dentária nas adolescentes com mães com Ensino Médio completo 
Características sociodemográficas de escolares do 9ำ ano do Ensino Fundamental, segundo o sexo. Capitais brasileiras, 2009 (prevalência e intervalos de $95 \%$ de confiança).

\begin{tabular}{|c|c|c|c|}
\hline & Masculino & Feminino & Total \\
\hline \multicolumn{4}{|l|}{ Idade (anos) } \\
\hline$\leq 13$ & $21,1(20,2-21,9)$ & $27,5(26,6-28,4)$ & $24,4(23,9-25,1)$ \\
\hline 14 & $45,8(44,8-46,9)$ & $48,3(47,3-49,2)$ & $47,2(46,4-47,8)$ \\
\hline 15 & $20,8(20,0-21,6)$ & $15,9(15,3-16,6)$ & $18,2(17,7-18,8)$ \\
\hline$\geq 16$ & $12,3(11,7-12,9)$ & $8,3(7,9-8,8)$ & $10,2(9,8-10,6)$ \\
\hline \multicolumn{4}{|l|}{ Raça/cor da pele } \\
\hline Branca & $41,7(40,6-42,7)$ & $38,8(37,9-39,7)$ & $40,2(39,5-40,8)$ \\
\hline Parda & $35,7(34,7-36,7)$ & $42,3(41,3-43,2)$ & $39,1(38,5-39,8)$ \\
\hline Preta & $14,9(14,2-15,6)$ & $11,0(10,5-11,6)$ & $12,9(12,4-13,3)$ \\
\hline Amarela & $3,4(3,1-3,8)$ & $4,0(3,7-4,4)$ & $3,7(3,5-4,0)$ \\
\hline Indígena & $4,3(4,0-4,8)$ & $3,9(3,6-4,2)$ & $4,1(3,9-4,4)$ \\
\hline \multicolumn{4}{|l|}{ Escola } \\
\hline Pública & $78,4(78,2-78,6)$ & $79,4(78,8-79,9)$ & $78,9(78,7-79,1)$ \\
\hline Privada & $21,6(21,4-21,8)$ & $20,6(20,1-21,2)$ & $21,1(20,9-21,3)$ \\
\hline \multicolumn{4}{|l|}{ Escolaridade da mãe } \\
\hline Sem estudo & $28,9(27,9-29,9)$ & $34,3(33,4-35,4)$ & $31,8(31,1-32,5)$ \\
\hline Ensino Fundamental completo & $17,2(16,4-18,1)$ & $16,6(15,8-17,3)$ & $16,9(16,3-17,4)$ \\
\hline Ensino Médio completo & $32,3(31,2-33,4)$ & $30,9(29,9-31,9)$ & $31,5(30,8-32,3)$ \\
\hline Ensino Superior completo & $21,6(20,8-22,5)$ & $18,2(17,4-18,9)$ & $19,8(19,3-20,4)$ \\
\hline \multicolumn{4}{|l|}{ Mora com o pai } \\
\hline $\operatorname{Sim}$ & $66,1(65,1-67,1)$ & $60,5(59,5-61,4)$ & $63,1(62,5-63,8)$ \\
\hline Não & $33,9(32,9-34,9)$ & $39,5(38,6-40,5)$ & $36,9(36,2-37,5)$ \\
\hline \multicolumn{4}{|l|}{ Mora com a mãe } \\
\hline $\operatorname{Sim}$ & $90,4(89,8-91,0)$ & $90,7(90,2-91,2)$ & $90,6(90,2-91,0)$ \\
\hline Não & $9,6(9,0-10,2)$ & $9,3(8,8-9,8)$ & $9,4(9,0-9,8)$ \\
\hline
\end{tabular}

(OR = 1,0; IC95\%: 1,07-3,36). A chance de fumar pelo menos 1 dia nos últimos 30 dias foi menor entre aqueles que escovaram os dentes 2 vezes ou mais por dia. Esta associação foi observada para o sexo masculino para as categorias de escolaridade materna "sem estudo" (OR = 0,59; IC95\%: 0,41-0,86) e "Ensino Superior" (OR = 0,56; IC95\%: 0,33-0,97), e para a categoria "sem estudo" para o sexo feminino (OR = 0,56; IC95\%: 0,38-0,83). O consumo de bebida alcoólica foi inversamente associado à escovação dentária para os adolescentes com mães sem estudo (OR = 0,66; IC95\%: 0,45-0,97). A chance de escovar os dentes 2 vezes ou mais por dia foi menor entre os adolescentes que relataram ter usado drogas no último mês. Esta associação foi encontrada para ambos os sexos e para todos os grupos de escolaridade materna, exceto para aqueles com escolaridade materna com Ensino Fundamental. A chance de praticar atividade física fora da escola foi maior entre adolescentes que escovaram os dentes 2 vezes ou mais por dia do sexo masculino, para as categorias de escolaridade materna sem estudo (OR = 1,57; IC95\%: 1,01-2,25) e com Ensino Médio (OR = 2,02; IC95\%: 1,39-2,95). A maior frequência do uso do cinto de segurança foi positivamente associada com a escovação dentária no sexo masculino para a escolaridade materna Ensino Médio (OR = 1,56; IC95\%: 1,01-2,42) e no sexo feminino para escolaridade materna sem estudo (OR = 2,13; IC95\%: 1,27-3,59).

\section{Discussão}

Modelos que buscam examinar mecanismos diretos e intermediários pelos quais fatores sociais, atitudinais e comportamentais produzem resultados em saúde bucal são relativamente escassos na literatura, embora alguns tenham sido propostos e testados para adultos 23 . Contudo, uma importante advertência é que modelos de inter- 
Comportamentos relacionados à saúde de escolares do 9o ano do Ensino Fundamental, segundo o sexo. Capitais brasileiras, 2009 (prevalência e intervalos de 95\% de confiança).

\begin{tabular}{|c|c|c|c|}
\hline & Masculino & Feminino & Total \\
\hline \multicolumn{4}{|l|}{ Escovação dentária } \\
\hline Não escova & $1,0(0,8-1,3)$ & $0,3(0,2-0,4)$ & $0,6(0,5-0,8)$ \\
\hline Uma vez ao dia & $5,1(4,6-5,5)$ & $3,5(3,1-3,8)$ & $4,2(3,9-4,5)$ \\
\hline Duas vezes ao dia & $24,1(23,2-25,0)$ & $19,3(18,6-20,1)$ & $21,6(21,0-22,2)$ \\
\hline Três vezes ao dia & $42,2(41,2-43,2)$ & $43,6(42,7-44,6)$ & $43,0(42,3-43,7)$ \\
\hline Quatro vezes ou mais ao dia & $27,6(26,7-28,6)$ & $33,3(32,4-34,2)$ & $30,6(30,0-31,3)$ \\
\hline \multicolumn{4}{|c|}{ Em quantos dias comeu doces nos últimos 7 dias } \\
\hline Nenhum & $10,9(10,2-11,5)$ & $5,4(5,1-5,8)$ & $8,0(7,6-8,4)$ \\
\hline 1 ou 2 & $25,4(24,6-26,3)$ & $18,6(17,9-19,4)$ & $21,9(21,3-22,4)$ \\
\hline 3 ou 4 & $21,1(20,3-22,0)$ & $17,6(16,9-18,3)$ & $19,3(18,7-19,8)$ \\
\hline 5 ou 6 & $15,7(15,0-16,5)$ & $16,9(16,1-17,6)$ & $16,3(15,8-16,9)$ \\
\hline Todos & $26,9(25,9-27,8)$ & $41,5(40,5-42,4)$ & $34,5(33,9-35,2)$ \\
\hline \multicolumn{4}{|c|}{ Número de dias que fumou nos últimos 30 dias } \\
\hline Nenhum & $80,5(79,7-81,3)$ & $79,4(78,5-80,1)$ & $79,9(79,3-80,4)$ \\
\hline Nenhum dia nos últimos 30 dias & $13,2(12,5-13,9)$ & $14,4(13,8-15,1)$ & $13,8(13,4-14,3)$ \\
\hline $1-5$ & $3,6(3,2-3,9)$ & $4,1(3,7-4,5)$ & $3,8(3,6-4,1)$ \\
\hline $6-19$ & $1,2(1,0-1,5)$ & $0,9(0,8-1,2)$ & $1,1(0,9-1,3)$ \\
\hline 20 ou mais & $1,5(1,3-1,8)$ & $1,2(1,0-1,5)$ & $1,4(1,2-1,6)$ \\
\hline \multicolumn{4}{|c|}{$\begin{array}{l}\text { Número de dias que tomou pelo menos um copo ou } \\
\text { dose de bebida alcoólica nos últimos } 30 \text { dias }\end{array}$} \\
\hline Nenhum & $73,5(72,6-74,4)$ & $71,9(71,0-72,8)$ & $72,7(72,0-73,3)$ \\
\hline $1-5$ & $20,0(19,2-20,8)$ & $22,3(21,5-23,2)$ & $21,2(20,6-21,8)$ \\
\hline $6-19$ & $4,5(4,1-5,0)$ & $4,4(4,0-4,8)$ & $4,4(4,2-4,7)$ \\
\hline $20-29$ & $0,9(0,7-1,1)$ & $0,9(0,8-1,1)$ & $0,9(0,8-1,1)$ \\
\hline Todos & $1,1(0,9-1,3)$ & $0,5(0,4-0,6)$ & $0,8(0,7-0,9)$ \\
\hline \multicolumn{4}{|c|}{ Quantas vezes você usou drogas nos últimos 30 dias } \\
\hline Nenhuma vez & $95,6(95,1-96,0)$ & $97,7(97,3-98,0)$ & $96,7(96,4-96,9)$ \\
\hline 1 ou 2 vezes & $2,3(2,0-2,6)$ & $1,6(1,3-1,9)$ & $1,9(1,7-2,1)$ \\
\hline 3 a 5 vezes & $0,6(0,5-0,8)$ & $0,3(0,2-0,4)$ & $0,5(0,4-0,6)$ \\
\hline 6 a 9 vezes & $0,5(0,3-0,7)$ & $0,2(0,1-0,3)$ & $0,3(0,2-0,4)$ \\
\hline 10 vezes ou mais & $1,0(0,8-1,3)$ & $0,2(0,1-0,3)$ & $0,6(0,5-0,7)$ \\
\hline \multicolumn{4}{|c|}{$\begin{array}{l}\text { Em quantos dias você fez atividade física fora da } \\
\text { escola nos últimos } 7 \text { dias }\end{array}$} \\
\hline Nenhum & $33,2(32,3-34,3)$ & $51,9(50,9-52,8)$ & $43,1(42,4-43,8)$ \\
\hline $1-2$ & $24,1(23,2-25,0)$ & $23,7(22,9-24,6)$ & $23,9(23,3-24,5)$ \\
\hline 3 & $11,9(11,2-12,6)$ & $8,2(7,7-8,8)$ & $10,0(9,6-10,4)$ \\
\hline 4 & $7,6(7,0-8,2)$ & $4,7(4,3-5,1)$ & $6,0(5,7-6,4)$ \\
\hline $5-7$ & $23,2(22,3-24,1)$ & $11,5(10,9-12,1)$ & $17,0(16,5-17,6)$ \\
\hline \multicolumn{4}{|c|}{$\begin{array}{l}\text { Número de vezes que usou o cinto de segurança no } \\
\text { carro nos últimos } 30 \text { dias * }\end{array}$} \\
\hline Nunca & $10,7(10,0-11,5)$ & $12,6(11,9-13,4)$ & $11,7(11,2-12,2)$ \\
\hline Raramente & $12,9(12,1-13,7)$ & $16,1(15,2-16,9)$ & $14,6(14,0-15,2)$ \\
\hline Às vezes & $11,0(10,3-11,9)$ & $11,0(10,3-11,7)$ & $11,0(10,5-11,5)$ \\
\hline Na maioria das vezes & $15,3(14,5-16,2)$ & $14,5(13,8-15,3)$ & $14,9(14,3-15,5)$ \\
\hline Sempre & $50,1(48,8-51,3)$ & $45,8(44,6-46,9)$ & $47,8(47,0-48,7)$ \\
\hline
\end{tabular}

* Dados para aqueles que relataram andar de carro. 
Comportamentos relacionados à saúde de escolares do sexo masculino do 9o ano do Ensino Fundamental, segundo condição socioeconômica (escolaridade materna). Capitais brasileiras, 2009 (prevalência e intervalos de 95\% de confiança).

\begin{tabular}{|c|c|c|c|c|c|}
\hline & \multicolumn{5}{|c|}{ Escolaridade materna } \\
\hline & Não estudou & Ensino & Ensino & Ensino & Total \\
\hline & & Fundamental & Médio & Superior & \\
\hline \multicolumn{6}{|l|}{ Escovação dentária (vezes por dia) } \\
\hline Menos de 2 & $7,2(6,2-8,5)$ & $5,7(4,5-7,2)$ & $5,6(4,7-6,6)$ & $5,4(4,4-6,5)$ & $6,0(5,5-6,6)$ \\
\hline 2 ou mais & $92,8(91,5-93,8)$ & $94,3(92,8-95,5)$ & $94,4(93,4-95,3)$ & $94,6(93,5-95,6)$ & $94,0(93,4-94,5)$ \\
\hline \multicolumn{6}{|c|}{ Em quantos dias comeu doces nos últimos 7 dias } \\
\hline Menos de 3 & $25,6(23,7-27,5)$ & $22,3(20,2-24,6)$ & $22,0(20,3-23,6)$ & $24,0(22,0-26,0)$ & $23,5(22,6-24,5)$ \\
\hline 3 ou mais & $74,4(72,5-76,3)$ & $77,7(75,4-79,8)$ & $78,0(76,4-79,7)$ & $76,0(74,0-78,0)$ & $76,5(75,5-77,4)$ \\
\hline \multicolumn{6}{|c|}{ Número de dias que fumou nos últimos 30 dias } \\
\hline Nenhum & $78,2(76,4-79,8)$ & $80,2(78,0-82,2)$ & $80,6(79,0-82,2)$ & $82,5(80,6-84,2)$ & $80,2(79,3-81,1)$ \\
\hline 1 ou mais & $21,8(20,2-23,6)$ & $19,8(17,8-22,0)$ & $19,4(17,8-21,0)$ & $17,5(15,8-19,4)$ & $19,8(18,9-20,7)$ \\
\hline \multicolumn{6}{|c|}{ Número de dias que tomou pelo menos um copo } \\
\hline \multicolumn{6}{|c|}{ ou dose de bebida alcoólica nos últimos 30 dias } \\
\hline Nenhum & $73,5(71,6-75,3)$ & $74,7(72,1-77,0)$ & $72,6(70,7-74,5)$ & $70,9(68,8-73,0)$ & $72,8(71,8-73,9)$ \\
\hline 1 ou mais & $26,5(24,7-28,4)$ & $25,3(23,0-27,9)$ & $27,4(25,5-29,3)$ & $29,1(27,0-31,2)$ & $27,2(26,1-28,2)$ \\
\hline \multicolumn{6}{|c|}{ Quantas vezes você usou drogas nos últimos 30 dias } \\
\hline Nenhum & $95,2(94,2-96,0)$ & $96,2(95,1-97,1)$ & $95,1(94,0-96,0)$ & $95,6(94,6-96,4)$ & $95,4(94,9-95,9)$ \\
\hline 1 ou mais & $4,8(4,0-5,8)$ & $3,8(2,9-4,9)$ & $4,9(4,0-6,0)$ & $4,4(3,6-5,4)$ & $4,6(4,1-5,1)$ \\
\hline \multicolumn{6}{|c|}{ Em quantos dias você fez atividade física fora } \\
\hline \multicolumn{6}{|l|}{ da escola nos últimos 7 dias } \\
\hline Nenhum & $34,8(32,8-36,9)$ & $33,9(31,3-36,5)$ & $32,7(30,7-34,6)$ & $24,6(22,6-26,7)$ & $31,7(30,7-32,8)$ \\
\hline 1 ou mais & $65,2(63,1-67,2)$ & $66,1(63,5-68,7)$ & $67,3(65,4-69,3)$ & $75,4(73,3-77,4)$ & $68,3(67,2-69,3)$ \\
\hline \multicolumn{6}{|c|}{ Número de vezes que usou o cinto de segurança } \\
\hline \multicolumn{6}{|l|}{ no carro nos últimos 30 dias * } \\
\hline Nunca, raramente ou às vezes & $39,0(36,3-41,8)$ & $40,9(37,6-44,3)$ & $33,6(31,4-35,9)$ & $25,7(23,6-27,8)$ & $33,8(32,6-35,1)$ \\
\hline Na maioria das vezes ou sempre & $61,0(58,2-63,7)$ & $59,1(55,7-62,4)$ & $66,4(64,1-68,6)$ & $74,3(72,2-76,4)$ & $66,2(64,9-67,4)$ \\
\hline
\end{tabular}

* Dados para aqueles que relataram andar de carro.

venção baseados em teorias psicológicas, cognitivas e comportamentais, desenvolvidas para adultos não são tão efetivos para adolescentes, que estão obviamente em estágios diferentes de desenvolvimento de suas capacidades.

Contexto de moradia e posição socioeconômica, ocupação e renda, grupo étnico e migração, além de sexo/gênero, são fatores que podem desempenhar um importante papel no processo saúde-doença 24,25,26,27,28. Não obstante, é preciso considerar conjuntamente os hábitos individuais, tais como o consumo precoce de tabaco e álcool, e padrões de higiene bucal 29,30,31 . Achados na literatura sugerem que adolescentes tabagistas são menos interessados em sua higiene bucal e na busca regular por cuidados odontológicos 32. Até mesmo fatores ligados a características físicas e o modo como estas são percebidas por ambos os sexos, tais como a al- tura e o peso, bem como a respectiva autoestima com a aparência, têm sido arrolados como possíveis correlatos da saúde bucal que variam no período transicional da adolescência 33,34,35,36.

É de particular importância para o contexto de análise adotado no presente trabalho a observação da possível relação entre comportamentos de higiene bucal e de higiene geral entre adolescentes. Há teorias que advogam a relação entre as convicções de saúde e os comportamentos de saúde dos indivíduos 37 . Nesta linha, sugere-se que convicções sobre saúde bucal podem mudar entre a adolescência e a maioridade, e, em particular, apontam-se possíveis associações entre convicções desfavoráveis progressivamente reguladas por perdas dentais devido à cárie, doença periodontal, pior higiene bucal, autoavaliação negativa de saúde bucal, e mais restaurações. Estudos revelam, ainda, que 
Comportamentos relacionados à saúde de escolares do sexo feminino do 9o ano do Ensino Fundamental, segundo condição socioeconômica (escolaridade materna). Capitais brasileiras, 2009 (prevalência e intervalos de 95\% de confiança).

\begin{tabular}{|c|c|c|c|c|c|}
\hline & \multicolumn{5}{|c|}{ Escolaridade materna } \\
\hline & Não estudou & Ensino & Ensino Médio & Ensino & Total \\
\hline & & Fundamental & & Superior & \\
\hline \multicolumn{6}{|l|}{ Escovação dentária (vezes por dia) } \\
\hline Menos de 2 & $4,7(4,0-5,6)$ & $3,4(2,6-4,5)$ & $2,9(2,4-3,6)$ & $3,0(2,3-3,9)$ & $3,6(3,3-4,1)$ \\
\hline 2 ou mais & $95,3(94,4-96,0)$ & $96,6(95,5-97,4)$ & $97,1(96,4-97,6)$ & $97,0(96,1-97,7)$ & $96,4(95,9-96,7)$ \\
\hline \multicolumn{6}{|c|}{ Em quantos dias comeu doces nos últimos 7 dias } \\
\hline Menos de 3 & $19,1(17,8-20,5)$ & $17,5(15,7-19,4)$ & $13,9(12,8-15,1)$ & $16,2(14,5-18,1)$ & $16,7(16,0-17,5)$ \\
\hline Três ou mais & $80,9(79,5-82,2)$ & $82,5(80,6-84,3)$ & $86,1(84,9-87,2)$ & $83,8(81,9-85,5)$ & $83,3(82,5-84,0)$ \\
\hline \multicolumn{6}{|c|}{ Número de dias que fumou nos últimos 30 dias } \\
\hline Nenhum & $76,1(74,5-77,6)$ & $79,3(77,2-81,3)$ & $80,3(78,7-81,8)$ & $83,9(81,9-85,7)$ & $79,4(78,5-80,2)$ \\
\hline 1 ou mais & $23,9(22,4-25,5)$ & $20,7(18,7-22,8)$ & $19,7(18,2-21,3)$ & $16,1(14,3-18,1)$ & $20,6(19,8-21,5)$ \\
\hline \multicolumn{6}{|c|}{ Número de dias que tomou pelo menos um copo } \\
\hline \multicolumn{6}{|c|}{ ou dose de bebida alcoólica nos últimos 30 dias } \\
\hline Nenhum & $70,7(69,0-72,3)$ & $74,1(71,9-76,3)$ & $71,5(69,7-73,2)$ & $70,0(67,6-72,2)$ & $71,4(70,4-72,3)$ \\
\hline 1 ou mais & $29,3(27,7-31,0)$ & $25,9(23,7-28,1)$ & $28,5(26,8-30,3)$ & $30,0(27,8-32,4)$ & $28,6(27,7-29,6)$ \\
\hline \multicolumn{6}{|c|}{ Quantas vezes você usou drogas nos últimos 30 dias } \\
\hline Nenhum & $97,2(96,4-97,8)$ & $97,9(97,0-98,5)$ & $97,8(97,1-98,3)$ & $97,9(97,1-98,5)$ & $97,6(97,3-97,9)$ \\
\hline 1 ou mais & $2,8(2,2-3,6)$ & $2,1(1,5-3,0)$ & $2,2(1,7-2,9)$ & $2,1(1,5-2,9)$ & $2,4(2,1-2,7)$ \\
\hline \multicolumn{6}{|c|}{ Em quantos dias você fez atividade física fora } \\
\hline \multicolumn{6}{|l|}{ da escola nos últimos 7 dias } \\
\hline Nenhum & $56,5(54,7-58,2)$ & $52,6(50,0-55,2)$ & $51,3(49,4-53,1)$ & $38,4(36,1-40,8)$ & $50,9(49,9-52,0)$ \\
\hline 1 ou mais & $43,5(41,8-45,3)$ & $47,4(44,8-50,0)$ & $48,7(46,9-50,6)$ & $61,6(59,2-63,9)$ & $49,1(48,0-50,1)$ \\
\hline \multicolumn{6}{|c|}{ Número de vezes que usou o cinto de segurança } \\
\hline \multicolumn{6}{|l|}{ no carro nos últimos 30 dias * } \\
\hline Nunca, raramente ou às vezes & $45,2(42,8-47,5)$ & $40,2(37,3-43,3)$ & $39,5(37,5-41,6)$ & $28,9(26,6-31,3)$ & $38,7(37,5-39,9)$ \\
\hline Na maioria das vezes ou sempre & $54,8(52,5-57,2)$ & $59,8(56,7-62,7)$ & $60,5(58,4-62,5)$ & $71,1(68,7-73,4)$ & $61,3(60,1-62,5)$ \\
\hline
\end{tabular}

* Dados para aqueles que relataram andar de carro.

a frequência de escovação dental é significativamente associada com fatores afins como, por exemplo, a higiene corporal rotineira (banhos) e a troca da roupa íntima 38 .

No presente estudo, a frequência de escovação dentária duas vezes ou mais ao dia foi de $95,2 \%$, sendo maior para o sexo feminino, o que se coaduna com a literatura em que se registram importantes diferenças em prevalências informadas sobre o hábito de escovar os dentes, entre países, para ambos os sexos. Amostras representativas de 32 países europeus e norte-americanos mostram variações de $16-80 \%$ para os meninos e 26-89\% para meninas. Gênero, afluência familiar e ocupação dos pais mostram-se significativamente associados com a frequência de escovação dental entre adolescentes 39 .

Neste trabalho algumas análises revelaram que a maior frequência de escovação dentária foi inversamente associada com a menor escolaridade materna, tanto no sexo masculino quanto no feminino, ou ainda, que o tabagismo diminuiu conforme o aumento da escolaridade materna. Estudos asseveram, por exemplo, que escovar os dentes duas vezes, diariamente, associa-se positivamente com fatores tais como o senso de coerência materna - um constructo central da teoria salutogênica e um recurso positivo para lidar com condições estressoras que alguns adolescentes apresentam 40 - ou, ainda, a possibilidade de coabitar com suas mães, mas negativamente com o hábito de fumar e com a vulnerabilidade à depressão 41.

Quando se trata de estudar a relação específica entre comportamentos de adolescentes e a saúde bucal, algumas abordagens buscam aumentar a efetividade das mudanças comportamentais positivas ${ }^{42}$. Não obstante, são estes 
Associação entre hábitos relacionados à saúde e condição socioeconômica (escolaridade materna) em escolares do 9o ano do Ensino Fundamental, segundo o sexo. Capitais brasileiras, 2009 (prevalência e intervalos de 95\% de confiança).

\begin{tabular}{|c|c|c|c|c|c|c|c|c|}
\hline & \multicolumn{4}{|c|}{ Masculino } & \multicolumn{4}{|c|}{ Feminino } \\
\hline & \multicolumn{4}{|c|}{ Escolaridade materna } & \multicolumn{4}{|c|}{ Escolaridade materna } \\
\hline & $\begin{array}{l}\text { Ensino } \\
\text { Superior }\end{array}$ & $\begin{array}{l}\text { Ensino } \\
\text { Médio }\end{array}$ & $\begin{array}{l}\text { Ensino } \\
\text { Fundamental }\end{array}$ & $\begin{array}{c}\text { Sem } \\
\text { estudo }\end{array}$ & $\begin{array}{l}\text { Ensino } \\
\text { Superior }\end{array}$ & $\begin{array}{l}\text { Ensino } \\
\text { Médio }\end{array}$ & $\begin{array}{l}\text { Ensino } \\
\text { Fundamental }\end{array}$ & $\begin{array}{c}\text { Sem } \\
\text { estudo }\end{array}$ \\
\hline $\begin{array}{l}\text { Escovação dentária: } 2 \text { vezes ou mais } \\
\text { por dia }\end{array}$ & 1,00 & $\begin{array}{c}0,96 \\
(0,72-1,23)\end{array}$ & $\begin{array}{c}0,94 \\
(0,68-1,31)\end{array}$ & $\begin{array}{c}0,73 \\
(0,55-0,96)\end{array}$ & 1,00 & $\begin{array}{c}1,02 \\
(0,72-1,44)\end{array}$ & $\begin{array}{c}0,88 \\
(0,58-1,31)\end{array}$ & $\begin{array}{c}0,62 \\
(0,45-0,87)\end{array}$ \\
\hline $\begin{array}{l}\text { Comeu doces } 3 \text { ou mais dias na } \\
\text { última semana }\end{array}$ & 1,00 & $\begin{array}{c}1,12 \\
(0,97-1,30)\end{array}$ & $\begin{array}{c}1,10 \\
(0,97-1,30)\end{array}$ & $\begin{array}{c}0,92 \\
(0,79-1,06)\end{array}$ & 1,00 & $\begin{array}{c}1,20 \\
(1,02-1,41)\end{array}$ & $\begin{array}{c}0,91 \\
(0,76-1,10)\end{array}$ & $\begin{array}{c}0,82 \\
(0,70-0,96)\end{array}$ \\
\hline $\begin{array}{l}\text { Fumou pelo menos } 1 \text { dia nos últimos } \\
30 \text { dias }\end{array}$ & 1,00 & $\begin{array}{c}1,13 \\
(0,96-1,33)\end{array}$ & $\begin{array}{c}1,16 \\
(0,97-1,39)\end{array}$ & $\begin{array}{c}1,32 \\
(1,13-1,54)\end{array}$ & 1,00 & $\begin{array}{c}1,28 \\
(1,07-1,52)\end{array}$ & $\begin{array}{c}1,36 \\
(1,13-1,64)\end{array}$ & $\begin{array}{c}1,64 \\
(1,39-1,93)\end{array}$ \\
\hline $\begin{array}{l}\text { Consumiu bebida alcoólica pelo } \\
\text { menos } 1 \text { dia nos últimos } 30 \text { dias }\end{array}$ & 1,00 & $\begin{array}{c}0,92 \\
(0,80-1,06)\end{array}$ & $\begin{array}{c}0,83 \\
(0,70-0,98)\end{array}$ & $\begin{array}{c}0,88 \\
(0,77-1,01)\end{array}$ & 1,00 & $\begin{array}{c}0,93 \\
(0,81-1,07)\end{array}$ & $\begin{array}{c}0,81 \\
(0,69-0,95)\end{array}$ & $\begin{array}{c}0,97 \\
(0,84-1,11)\end{array}$ \\
\hline $\begin{array}{l}\text { Usou drogas pelo menos } 1 \text { dia nos } \\
\text { últimos } 30 \text { dias }\end{array}$ & 1,00 & $\begin{array}{c}1,13 \\
(0,83-1,52)\end{array}$ & $\begin{array}{c}0,86 \\
(0,61-1,21)\end{array}$ & $\begin{array}{c}1,11 \\
(0,83-1,49)\end{array}$ & 1,00 & $\begin{array}{c}1,04 \\
(0,69-1,59)\end{array}$ & $\begin{array}{c}1,00 \\
(0,61-1,61)\end{array}$ & $\begin{array}{c}1,35 \\
(0,90-2,01)\end{array}$ \\
\hline $\begin{array}{l}\text { Praticou pelo menos } 1 \text { dia de } \\
\text { atividade física fora da escola nos } \\
\text { últimos } 7 \text { dias }\end{array}$ & 1,00 & $\begin{array}{c}0,67 \\
(0,58-0,77)\end{array}$ & $\begin{array}{c}0,64 \\
(0,54-0,75)\end{array}$ & $\begin{array}{c}0,61 \\
(0,53-0,70)\end{array}$ & 1,00 & $\begin{array}{c}0,59 \\
(0,52-0,67)\end{array}$ & $\begin{array}{c}0,56 \\
(0,49-0,65)\end{array}$ & $\begin{array}{c}0,48 \\
(0,45-0,55)\end{array}$ \\
\hline $\begin{array}{l}\text { Usou cinto de segurança na maioria } \\
\text { das vezes ou sempre nos últimos } 30 \\
\text { dias }\end{array}$ & 1,00 & $\begin{array}{c}0,68 \\
(0,59-0,79)\end{array}$ & $\begin{array}{c}0,50 \\
(0,42-0,60)\end{array}$ & $\begin{array}{c}0,54 \\
(0,46-0,63)\end{array}$ & 1,00 & $\begin{array}{c}0,62 \\
(0,54-0,72)\end{array}$ & $\begin{array}{c}0,60 \\
(0,51-0,71)\end{array}$ & $\begin{array}{c}0,49 \\
(0,42-0,57)\end{array}$ \\
\hline
\end{tabular}

Associação entre hábitos relacionados à saúde e escovação dentária 2 vezes ou mais por dia em escolares do 9o ano do Ensino Fundamental, segundo condição socioeconômica (escolaridade materna) e sexo. Capitais brasileiras, 2009 (prevalência e intervalos de $95 \%$ de confiança).

\begin{tabular}{|c|c|c|c|c|c|c|c|c|}
\hline & \multicolumn{4}{|c|}{ Masculino } & \multicolumn{4}{|c|}{ Feminino } \\
\hline & \multicolumn{4}{|c|}{ Escolaridade materna } & \multicolumn{4}{|c|}{ Escolaridade materna } \\
\hline & $\begin{array}{l}\text { Sem } \\
\text { estudo }\end{array}$ & $\begin{array}{l}\text { Ensino } \\
\text { Fundamental }\end{array}$ & $\begin{array}{l}\text { Ensino } \\
\text { Médio }\end{array}$ & $\begin{array}{l}\text { Ensino } \\
\text { Superior }\end{array}$ & $\begin{array}{l}\text { Sem } \\
\text { estudo }\end{array}$ & $\begin{array}{l}\text { Ensino } \\
\text { Fundamental }\end{array}$ & $\begin{array}{l}\text { Ensino } \\
\text { Médio }\end{array}$ & $\begin{array}{l}\text { Ensino } \\
\text { Superior }\end{array}$ \\
\hline $\begin{array}{l}\text { Comeu doces } 3 \text { ou mais dias na última } \\
\text { semana }\end{array}$ & $\begin{array}{c}1,34 \\
(0,89-2,01)\end{array}$ & $\begin{array}{c}1,22 \\
(0,69-2,14)\end{array}$ & $\begin{array}{c}0,82 \\
(0,55-1,33)\end{array}$ & $\begin{array}{c}1,11 \\
(0,68-1,79)\end{array}$ & $\begin{array}{c}1,48 \\
(0,99-2,22)\end{array}$ & $\begin{array}{c}0,71 \\
(0,41-1,25)\end{array}$ & $\begin{array}{c}1,90 \\
(1,07-3,36)\end{array}$ & $\begin{array}{c}0,88 \\
(0,42-1,82)\end{array}$ \\
\hline $\begin{array}{l}\text { Fumou pelo menos } 1 \text { dia nos últimos } \\
30 \text { dias }\end{array}$ & $\begin{array}{c}0,59 \\
(0,41-0,86)\end{array}$ & $\begin{array}{c}1,07 \\
(0,63-1,81)\end{array}$ & $\begin{array}{c}0,80 \\
(0,52-1,23)\end{array}$ & $\begin{array}{c}0,56 \\
(0,33-0,97)\end{array}$ & $\begin{array}{c}0,56 \\
(0,38-0,83)\end{array}$ & $\begin{array}{c}1,37 \\
(0,67-2,78)\end{array}$ & $\begin{array}{c}0,75 \\
(0,46-1,23)\end{array}$ & $\begin{array}{c}0,59 \\
(0,29-1,19)\end{array}$ \\
\hline $\begin{array}{l}\text { Consumiu bebida alcoólica pelo } \\
\text { menos } 1 \text { dia nos últimos } 30 \text { dias }\end{array}$ & $\begin{array}{c}0,66 \\
(0,45-0,97)\end{array}$ & $\begin{array}{c}0,71 \\
(0,42-1,22)\end{array}$ & $\begin{array}{c}0,78 \\
(0,52-1,17)\end{array}$ & $\begin{array}{c}0,80 \\
(0,49-1,28)\end{array}$ & $\begin{array}{c}1,04 \\
(0,69-1,57)\end{array}$ & $\begin{array}{c}0,84 \\
(0,41-1,69)\end{array}$ & $\begin{array}{c}0,79 \\
(0,48-1,31)\end{array}$ & $\begin{array}{c}0,83 \\
(0,46-1,49)\end{array}$ \\
\hline $\begin{array}{l}\text { Usou drogas pelo menos } 1 \text { dia nos } \\
\text { últimos } 30 \text { dias }\end{array}$ & $\begin{array}{c}0,31 \\
(0,17-0,58)\end{array}$ & $\begin{array}{c}0,32 \\
(0,14-0,71)\end{array}$ & $\begin{array}{c}0,55 \\
(0,27-1,13)\end{array}$ & $\begin{array}{c}0,36 \\
(0,17-0,80)\end{array}$ & $\begin{array}{c}0,62 \\
(0,22-1,73)\end{array}$ & $\begin{array}{c}0,25 \\
(0,07-0,92)\end{array}$ & $\begin{array}{c}0,21 \\
(0,07-0,63)\end{array}$ & $\begin{array}{c}0,38 \\
(0,09-1,64)\end{array}$ \\
\hline $\begin{array}{l}\text { Praticou pelo menos } 1 \text { dia de atividade } \\
\text { física fora da escola nos últimos } 7 \text { dias }\end{array}$ & $\begin{array}{c}1,57 \\
(1,01-2,25)\end{array}$ & $\begin{array}{c}1,01 \\
(0,62-1,66)\end{array}$ & $\begin{array}{c}2,02 \\
(1,39-2,95)\end{array}$ & $\begin{array}{c}1,25 \\
(0,84-1,86)\end{array}$ & $\begin{array}{c}1,31 \\
(0,90-1,91)\end{array}$ & $\begin{array}{c}1,08 \\
(0,59-1,97)\end{array}$ & $\begin{array}{c}1,12 \\
(0,72-1,74)\end{array}$ & $\begin{array}{c}1,63 \\
(0,93-2,87)\end{array}$ \\
\hline $\begin{array}{l}\text { Usou cinto de segurança na maioria } \\
\text { das vezes ou sempre nos últimos } 30 \\
\text { dias }\end{array}$ & $\begin{array}{c}1,54 \\
(0,96-2,46)\end{array}$ & $\begin{array}{c}1,77 \\
(0,93-3,36)\end{array}$ & $\begin{array}{c}1,56 \\
(1,01-2,42)\end{array}$ & $\begin{array}{c}1,27 \\
(0,81-1,99)\end{array}$ & $\begin{array}{c}2,13 \\
(1,27-3,59)\end{array}$ & $\begin{array}{c}1,13 \\
(0,58-2,19)\end{array}$ & $\begin{array}{c}1,30 \\
(0,77-2,18)\end{array}$ & $\begin{array}{c}1,05 \\
(0,58-1,92)\end{array}$ \\
\hline
\end{tabular}


mesmos autores os primeiros a reconhecer um limitado sucesso das intervenções que visam mudanças comportamentais em adolescentes, dificultando a identificação dos melhores modos para promover saúde bucal para este grupo populacional ${ }^{42}$. Neste aspecto é importante lembrar que a adolescência é o período da vida em que se acentua a ocorrência de várias doenças e agravos em saúde bucal, particularmente para os subgrupos mais fragilizados desta faixa etária, em função de determinantes socioeconômicos, étnicos, de gênero, de geração, dentre outros: cárie dentária, periodontopatias, maloclusões, traumatismos, doenças sexualmente transmissíveis com manifestações bucais, lesões com potencial de malignidade, dores de origem odontogênica, perdas dentárias, dentre outras condições tornam-se mais frequentes e mais graves $42,43,44,45,46,47,48,49$.

A PeNSE obteve informações nacionais sobre adolescentes e o uso da base de dados por ela gerada permitiu que o presente artigo identificasse a associação entre alguns comportamentos relacionados à saúde bucal dos adolescentes e certas condições sociais de suas famílias, particularmente a escolaridade das mães. Um aspecto positivo a ser ressaltado em relação a PeNSE é sua representatividade nacional, assim como o fato de a amostra contemplar a participação de escolas públicas e privadas de todas as capitais do país e Distrito Federal. Como limitação do estudo pode ser citado o fato de o questionário utilizado na pesquisa ter sido autoaplicado, o que pode gerar erros de aferição por sub-relato ou mesmo dificuldades na compreensão das questões pelos adolescentes.

Intervenções planejadas para promover a saúde ou, eventualmente, mudar o comportamento de higiene bucal de adolescentes parecem ser mais efetivas se levam em consideração os modos habituais de "levar a vida" deste grupo populacional, ou seja, suas rotinas mentais e comportamentais 50 . Ainda, é preciso considerar que a predisposição psicológica e o ambiente familiar podem influenciar o comportamento de higiene bucal significativamente.

Os resultados revelam a associação entre a saúde bucal e os comportamentos de risco à saúde dos adolescentes, mediados por sua condição de vida. Portanto, ações relacionadas a melhorias no ambiente que cerca o adolescente certamente produzirão um efeito positivo nos aspectos de bem-estar e de saúde destes e de suas famílias. É particularmente importante ressaltar, portanto, que o ambiente escolar seja um espaço promotor da saúde bucal.

\footnotetext{
Resumo

Investigou-se a associação entre comportamentos relacionados à saúde bucal e geral e condição socioeconômica, e a relação entre comportamentos relacionados à saúde e escovação dentária em adolescentes. A base de dados utilizada foi da Pesquisa Nacional de Saúde do Escolar (PeNSE), um estudo transversal de base populacional realizado em 2009 com alunos das 27 capitais brasileiras. Informações sociodemográficas e comportamentos relacionados à saúde foram coletados. $O$ estudo envolveu 49.189 adolescentes (47,5\% do sexo masculino), sendo a maioria com 14 anos de idade e de escola pública. A frequência de escovação dentária
}

assim como outros comportamentos relacionados à saúde se associou com a condição socioeconômica de maneira distinta entre sexos. Foram observadas associações entre hábitos relacionados à saúde e a frequência de escovação dentária em ambos os sexos, porém com variações conforme a condição socioeconômica. O planejamento de intervenções para promoção da saúde de adolescentes deve levar em conta suas características individuais, o contexto familiar e social.

Comportamento do Adolescente; Escovação Dentária; Higiene Bucal; Saúde Bucal 


\section{Colaboradores}

M. V. Vettore contribuiu com a concepção do estudo, análise e interpretação dos dados e redação do artigo. S. J. Moysés contribuiu com a concepção do estudo, revisão crítica da literatura, interpretação dos dados e redação do artigo. L. M. V. Sardinha contribuiu com a redação do artigo. B. P. M. Iser contribuiu com a concepção do estudo, revisão dos dados e redação do artigo.

\section{Referências}

1. Zwig JM, Phillips SD, Lindberg LD. Predicting adolescent profiles of risk: looking beyond demographics. J Adolesc Health 2002; 31:343-53.

2. Hublet A, De Bacquer D, Valimaa R, Godeau E, Schmid H, Rahav G, et al. Smoking trends among adolescents from 1990 to 2002 in ten European countries and Canada. BMC Public Health 2006; 6:280.

3. Alamian A, Paradis G. Correlates of multiple chronic disease behavioral risk factors in Canadian children and adolescents. Am J Epidemiol 2009; 170:1279-89.

4. Lynch JW, Kaplan GA, Salonen JT. Why do poor people behave poorly? Variation in adult health behaviours and psychosocial characteristics by stages of the socioeconomic lifecourse. Soc Sci Med 1997; 44:809-19.

5. Nicolau B, Marcenes W, Bartley M, Sheiham A. Associations between socio-economic circumstances at two stages of life and adolescents' oral health status. J Public Health Dent 2005; 65:14-20.
6. Petersen PE. Sociobehavioural risk factors in dental caries: international perspectives. Community Dent Oral Epidemiol 2005; 33:274-9.

7. Sanders AE, Spencer AJ, Stewart JF. Clustering of risk behaviours for oral and general health. Community Dent Health 2005; 22:133-40.

8. Sabbah W, Tsakos G, Chandola T, Sheiham A, Watt RG. Social gradients in oral and general health. J Dent Res 2007; 86:992-6.

9. Koivusilta L, Rimpela A, Vikat A. Health behaviours and health in adolescence as predictors of educational level in adulthood: a follow-up study from Finland. Soc Sci Med 2003; 57:577-93.

10. Ostberg AL, Lindblad U, Halling A. Self-perceived oral health in adolescents associated with family characteristics and parental employment status. Community Dent Health 2003; 20:159-64. 
11. Peres MA, Peres KG, Barros AJ, Victora CG. The relation between family socioeconomic trajectories from childhood to adolescence and dental caries and associated oral behaviours. J Epidemiol Community Health 2007; 61:141-5.

12. Kawamura M, Takase N, Sasahara H, Okada M. Teenagers' oral health attitudes and behavior in Japan: comparison by sex and age group. J Oral Sci 2008; 50:167-74.

13. Petersen PE, Jiang H, Peng B, Tai BJ, Bian Z. Oral and general health behaviours among Chinese urban adolescents. Community Dent Oral Epidemiol 2008; 36:76-84.

14. Davoglio RS, Aerts DR, Abegg C, Freddo SL, Monteiro L. Factors associated with oral health habits and use of dental services by adolescents. Cad Saúde Pública 2009; 25:655-67.

15. Nicolau B, Marcenes W, Bartley M, Sheiham A. A life course approach to assessing causes of dental caries experience: the relationship between biological, behavioural, socio-economic and psychological conditions and caries in adolescents. Caries Res 2003; 37:319-26.

16. Peres KG, Peres MA, Araujo CL, Menezes AM, Hallal PC. Social and dental status along the life course and oral health impacts in adolescents: a population-based birth cohort. Health Qual Life Outcomes 2009; 7:95.

17. Moysés ST, Moysés SJ, Watt RG, Sheiham A. Associations between health promoting schools policies on some indicators of oral health. Health Promot Int 2003; 18:209-18.

18. Instituto Brasileiro de Geografia e Estatística. Pesquisa Nacional por Amostra de Domicílios 2008. v. 29. http://www.ibge.gov.br/home/estatistica/ populacao/trabalhoerendimento/pnad2008/bra silpnad2008.pdf (acessado em 15/Nov/2010).

19. Eaton DK, Kann L, Kinchen S, Shanklin S, Ross J, Hawkins J, et al. Youth risk behavior surveillance, United States, 2007. http://www.cdc.gov/mmwr/ PDF/ss/ss5704.pdf (acessado em Dez/2009).

20. World Health Organization. Inequalities young people's health: key findings from the Health Behaviour in School-aged Children (HBSC) 2005/2006 survey fact sheet. http://www.euro.who.int/Docu ment/Mediacentre/fs_hbsc_17june2008_e.pdf (acessado em Dez/2009).

21. Instituto Brasileiro de Geografia e Estatística. Pesquisa Nacional de Saúde do Escolar (PeNSE) 2009. http://www.ibge.gov.br/home/estatistica/popula cao/pense/pense.pdf (acessado em 15/Nov/2010).

22. Vigo Á. Modelando desfechos comuns: viés e precisão. Cad Saúde Pública 2006; 22:2496-7.

23. Baker SR. Applying Andersen's behavioural model to oral health: what are the contextual factors shaping perceived oral health outcomes? Community Dent Oral Epidemiol 2009; 37:485-94.

24. Kallestal C, Fjelddahl A. A four-year cohort study of caries and its risk factors in adolescents with high and low risk at baseline. Swed Dent J 2007; 31:11-25.

25. Pattussi MP, Olinto MT, Hardy R, Sheiham A. Clinical, social and psychosocial factors associated with self-rated oral health in Brazilian adolescents. Community Dent Oral Epidemiol 2007; 35:377-86.
26. De Reu G, Vanobbergen J, Martens LC. The influence of social indices on oral health and oral health behaviour in a group of Flemish socially deprived adolescents. Community Dent Health 2008; 25:33-7.

27. Freddo SL, Aerts DR, Abegg C, Davoglio R, Vieira PC, Monteiro L. Hábitos de higiene bucal e utilização de serviços odontológicos em escolares de uma cidade da Região Sul do Brasil. Cad Saúde Pública 2008; 24:1991-2000.

28. Kanli A, Kanbur NO, Dural S, Derman O. Effects of oral health behaviors and socioeconomic factors on a group of Turkish adolescents. Quintessence Int 2008; 39:e26-32.

29. Heikkinen AM, Pajukanta R, Pitkaniemi J, Broms U, Sorsa T, Koskenvuo M, et al. The effect of smoking on periodontal health of 15- to 16-year-old adolescents. J Periodontol 2008; 79:2042-7.

30. Strauch ES, Pinheiro RT, Silva RA, Horta BL. Uso de álcool por adolescentes: estudo de base populacional. Rev Saúde Pública 2009; 43:647-55.

31. Ostberg AL, Ericsson JS, Wennström JL, Abrahamsson KH. Socio-economic and lifestyle factors in relation to priority of dental care in a Swedish adolescent population. Swed Dent J 2010; 34:87-94.

32. Hellqvist L, Rolandsson M, Birkhed D, Hugoson A. Tobacco use in relation to socioeconomic factors and dental care habits among Swedish individuals 15-70 years of age, 1983-2003. Int J Dent Hyg 2009; 7:62-70.

33. Kallestal C, Dahlgren L, Stenlund H. Oral health behavior and self-esteem in Swedish adolescents over four years. J Adolesc Health 2006; 38:583-90.

34. Kantovitz KR, Pascon FM, Rontani RM, Gavião MB. Obesity and dental caries: a systematic review. Oral Health Prev Dent 2006; 4:137-44.

35. Koerber A, Graumlich S, Punwani IC, Berbaum ML, Burns JL, Levy SR, et al. Covariates of tooth-brushing frequency in low-income African Americans from grades 5 to 8. Pediatr Dent 2006; 28:524-30.

36. Freire MC, Sheiham A, Netuveli G. Relationship between height and dental caries in adolescents. Caries Res 2008; 42:134-40.

37. Broadbent JM, Thomson WM, Poulton R. Oral health beliefs in adolescence and oral health in young adulthood. J Dent Res 2006; 85:339-43.

38. Dorri M, Sheiham A, Watt RG. Relationship between general hygiene behaviours and oral hygiene behaviours in Iranian adolescents. Eur J Oral Sci 2009; 117:407-12.

39. Maes L, Vereecken C, Vanobbergen J, Honkala S. Tooth brushing and social characteristics of families in 32 countries. Int Dent J 2006; 56:159-67.

40. Freire M, Hardy R, Sheiham A. Mothers' sense of coherence and their adolescent children's oral health status and behaviours. Community Dent Health 2002; 19:24-31.

41. Ayo-Yusuf OA, Reddy PS, Van Den Borne BW. Longitudinal association of adolescents' sense of coherence with tooth-brushing using an integrated behaviour change model. Community Dent Oral Epidemiol 2009; 37:68-77.

42. Brukiene V, Aleksejuniene J. Theory-based oral health education in adolescents. Stomatologija 2010; 12:3-9. 
43. Goes PS, Watt R, Hardy RG, Sheiham A. The prevalence and severity of dental pain in 14-15 year old Brazilian schoolchildren. Community Dent Health 2007; 24:217-24.

44. Fadavi S, Sevandal MC, Koerber A, Punwani I. Survey of oral health knowledge and behavior of pregnant minority adolescents. Pediatr Dent 2009; 31:405-8.

45. Lopez R, Frydenberg M, Baelum V. Contextual effects in the occurrence of periodontal attachment loss and necrotizing gingival lesions among adolescents. Eur J Oral Sci 2009; 117:547-54.

46. Muirhead V, Quinonez C, Figueiredo R, Locker D. Oral health disparities and food insecurity in working poor Canadians. Community Dent Oral Epidemiol 2009; 37:294-304.

47. Noro LR, Roncalli AG, Mendes Júnior FI, Lima KC. Incidência de cárie dentária em adolescentes em município do Nordeste brasileiro, 2006. Cad Saúde Pública 2009; 25:783-90.
48. Braveman PA, Cubbin C, Egerter S, Williams DR, Pamuk E. Socioeconomic disparities in health in the United States: what the patterns tell us. Am J Public Health 2010; 100 Suppl 1:S185-96.

49. Carvalho ML, Moysés SJ, Bueno RE, Shimakura S, Moysés ST. A geographical population analysis of dental trauma in school-children aged 12 and 15 in the city of Curitiba-Brazil. BMC Health Serv Res 2010; 10:203.

50. Schüz B, Wiedemann AU, Mallach N, Scholz U. Effects of a short behavioural intervention for dental flossing: randomized-controlled trial on planning when, where and how. J Clin Periodontol 2009; 36:498-505.

Recebido em 17/Fev/2011

Versão final reapresentada em $02 / \mathrm{Jul} / 2011$

Aprovado em 12/Jul/2011 